\title{
User Authentication Platform using Provisioning in Cloud Computing Environment
}

\author{
Hyosik Ahn, Hyokyung Chang, Changbok Jang, Euiin Choi* \\ Dept. Of Computer Engineering, Hannam University, Daejeon, Korea \\ \{hsahn, hkjang, chbjang\}@dblab.hannam.ac.kr, eichoi@hnu.kr
}

\begin{abstract}
Cloud computing is a computing environment centered on users and can use programs or documents stored respectivily in servers by operating an applied software such as Web browser through diverse devices on where users can access Internet as an on-demand outsourcing service of IT resources using Internet. In order to use these cloud computing service, a user authentication is needed. This paper proposed the platform for user authentication using provisioning in Cloud computing environment.
\end{abstract}

Keywords: Cloud Computing, User Authentication, Provisioning, Security

\section{Introduction}

Gartner announced Top 10 of IT strategy technologies of the year of 2010 in 2009, October. Strategy technologies Gartner mentioned are the technologies which importantly affect enterprises for the next 3 years and have a powerful effect on IT and business. They may affect long-term plans, programs, and major projects of enterprises and help enterprises get strategic advantages if enterprises adopt them a head start. Cloud Computing got the top rank (2nd rank in 2009)[1][2]. Cloud Computing is a model of performance business and also infrastructure management methodology. It lets users use hardware, software and network resources as much as possible so as to provide innovative services through Web in these business performance models and also enables to provision a server according to needs of the logical by using automated advanced tools[3][4].

Also, Cloud Computing models offer both users and IT managers user interface which helps manage provisioned resources easy through the entire life cycle of a service request[5][6]. When resources requested by the user arrives through Cloud, the user can track the order consisted of a certain number of servers and software and operate jobs such as checking the state of the resources provided, adding a server, changing the installed software, removing a server, increasing or decreasing allocated processing power and memory or storage, even starting, aborting, and restarting server[7].

\footnotetext{
* Corresponding Author
} 
However, the diffusion of cloud computing incites users' desires for more improved, faster and more secure service delivery. Hence, security issues in the Cloud Computing environment are constantly emerging and authentication and access control has been studying. A user in the Cloud Computing environment has to complete the personal authentication process required by the service provider whenever using a new Cloud service. In this process, in the case that the characteristic and safety have been invaded by any attack, there will be a severe damage because personal information stored in the database and business processing service have been exposed or information related to individuals or organizations will be exposed as well.

Therefore, this paper designs a platform for user authentication using provisioning in the Cloud Computing environment.

\section{Related Works}

\subsection{Definition of Cloud computing and the Appearance Background}

Cloud Computing is a kind of on-demand computing method that lets users use IT resources such as network, server, storage, service, application, and so on via Internet when needing them rather than owning them[5]. Cloud Computing can be considered as a sum of SaaS (Software as a Service) and utility computing and Figure 1 shows the roles of users or providers in the Cloud Computing under the concept[9].

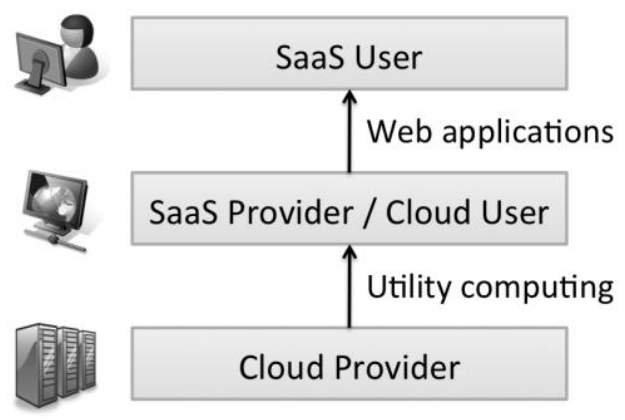

Figure 1. Users and Providers of Cloud Computing

\subsection{Provisioning}

Provisioning is a procedure and behavior that prepares required knowledge in advance and provides by requests in order to find the best thing. That is, it allocates, deploys, 
and distributes infrastructure resources to meet the needs of users or business and helps use the resources in the system[9].

- Server Resources Provisioning: prepares resources like the CPU of the server and memory by allocating and placing the resources appropriately

- OS Provisioning: prepares OS operative by installing OS in the server and configuring tasks

- Software Provisioning: prepares to operate by installing/distributing Software(WAS, DBMS, Application, etc.) in the system and doing configuration settings needed

- Storage Provisioning: enables to identify wasted or unused storage and put it into a common pool. If there is a request of storage since then, the administrator gets one out of the pool and uses. Possible to construct infrastructure to heighten the efficiency of storage

- Account Provisioning: the process that HR manager and IT manager take the appropriate approval process and then generate accounts needed for various applications such as e-mail. groupware, ERP, etc. or change the access authorities when the category of the resources that users access changes[10]

Figure 2 shows such a provisioning service.

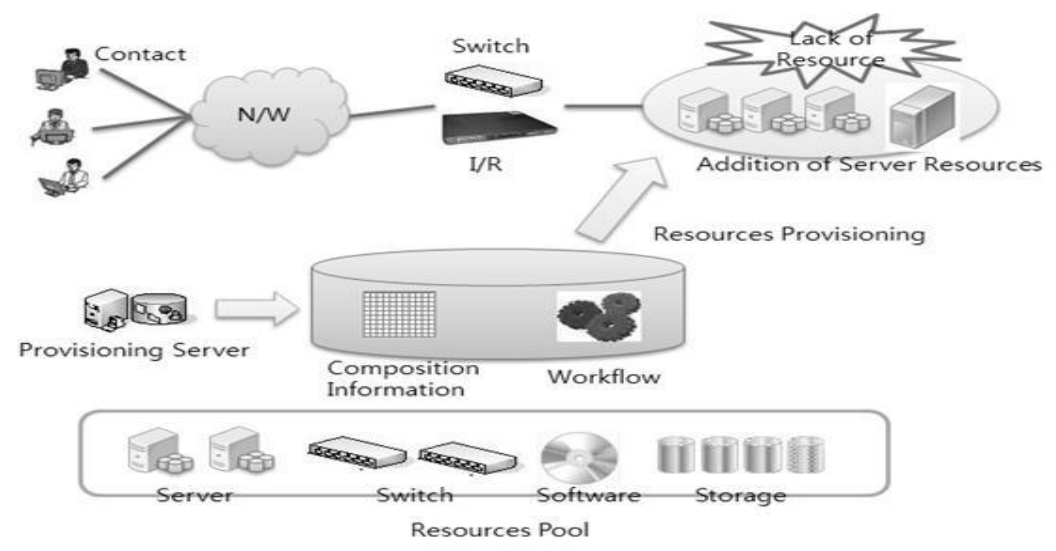

Figure 2. Provisioning Service

\subsection{Security Technology in the Cloud computing Environment}

There are no concrete security technologies in Cloud Computing, however, if we regard Cloud Computing as an extension of the existing IT technologies, it is possible to divide some of them by each component of Cloud Computing and apply to[12]. Access control and user authentication are representative as security technologies used for platforms. Access control is the technology that controls a process in the operating system not to approach the area of another process. There are DAC 
(Discretionary Access Control), MAC (Media Access Control), and RBAC (RoleBased Access Control). DAC helps a user establish the access authority to the resources that he/she owns as he/she pleases. MAC establishes the vertical/horizontal access rules in the system at the standard of security level and area for the resources and uses them. RBAC gives an access authority to a user group based on the role the user group plays in the organization, not to a user. RBAC is widely used because it is fit for the commercial organizations. Technologies used to authenticate a user are Id/password, Public Key Infrastructure, multi-factor authentication, SSO (Single Sign On), MTM (Mobile Trusted Module), and i-Pin[11].

\section{User Authentication in the Cloud computing Environment}

\subsection{User Authentication Technology in the Cloud computing Environment}

Users in the Cloud Computing environment have to complete the user authentication process required by the service provider whenever they use new Cloud service. Generally a user registers with offering personal information and a service provider provides a user's own ID (identification) and an authentication method for user authentication after a registration is done. Then the user uses the ID and the authentication method to operate the user authentication when the user accesses to use a Cloud Computing service[13]. Unfortunately, there is a possibility that the characteristics and safety of authentication method can be invaded by an attack during the process of authentication, and then it could cause severe damages. Hence, there must be not only security but also interoperability for user authentication of Cloud Computing.

\subsection{Weakness of User Authentication Technology in Cloud computing}

The representative user authentication security technologies described above have some weaknesses[11][12].

- Id/password: the representative user authentication method. It is simple and easy to use, but it has to have a certain level of complication and regular renewal to keep the security.

- PKI(Public Key Infrastructure): an authentication means using a public-key cryptography. It enables to authenticate the other party based on the certificate without shared secret information. In PKI structure, it is impossible to manage and inspect the process of client side.

- Multi-factor: a method to heighten the security intensity by combining a few means of authentication. Id, password, biometrics like fingerprint and iris, certificate, OTP (One Tome Pad), etc. are used. OTP information as well as $\mathrm{Id} /$ password can be disclosed to an attacker. 
- SSO (Single Sign On): a kind of passport if it gets authentication from a site, then it can go through to other sites with assertion and has no authentication process. The representative standard of assertion is SAML.

- MTM (Mobile Trusted Module): a hardware-based security module. It is a proposed standard by TCG (Trusted Computing Group) which Nokia, Samsung, France Telecom, Ericson, etc. take part in. It is mainly applied to authenticate terminals from telecommunications, however, it is being considered as a Cloud Computing authentication method with SIM (Subscriber Identity Module) because of generalization of smartphone[15].

- i-Pin: a technique to use to confirm a user's identification when the user uses Internet in Korea now. It operates in a way that the organization which itself performed the identification of the user issues the assertion.

\section{Platform Design for user authentication using Provisioning in Cloud Computing Environment}

\subsection{User Authentication using Provisioning}

User authentication platform using provisioning first authenticates by using ID/Password, PKI, SSO, etc. which a user input. Second, it authenticates with Authentication Manager through the user profile and patterns and stores the changes of the state via Monitor. When using Cloud Computing services, to solve the inconvenience of user authentication, user's information is stored in the User Information. Figure 3 shows a conceptual architecture.

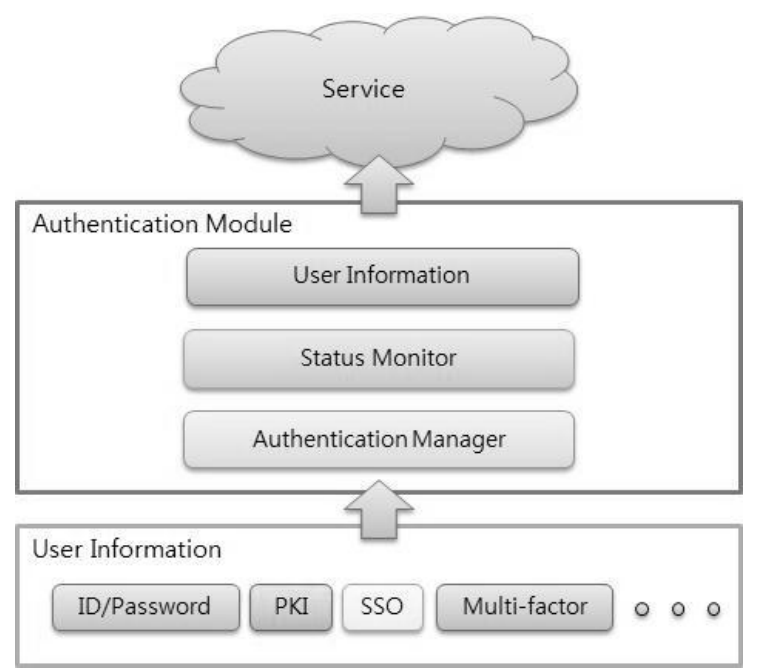

Figure 3. Conceptual Architecture 
For user authentication, Authentication Module has the previous user profile and log data record by Provisioning strategy.

\subsection{Design of User Authentication Platform Using Provisioning}

Figure 4 shows the composition of User authentication platform using provisioning proposed in this paper.

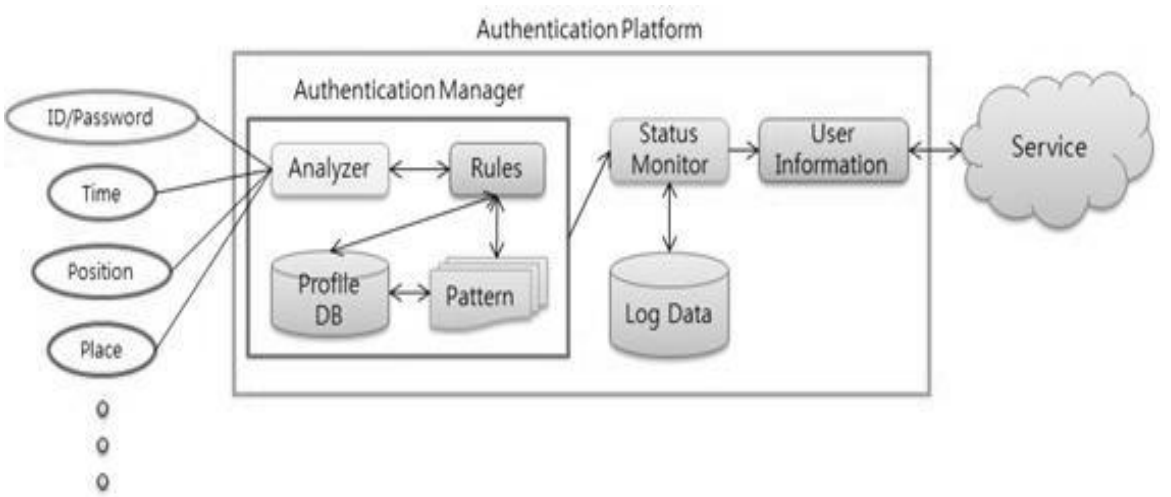

Figure 4. Authentication Platform

To authenticate a user first, Analyzer analyzes information such as ID/Password, Time, Position, Place, etc. it analyzes user pattern by using Rules for user authentication. To analyze user pattern, it authenticates a user by analyzing with current status and Rules of Profile DB. Profile DB stores user profile like existing user login time, location, position, etc. Also, it monitors the changes of user's status and situations via Status Monitor, records them in the database, and puts user information into User Information. That is how the user authentication process which the service provider asks to users whenever they use Cloud Computing services.

\section{Conclusions}

In this paper, user authentication platform using Provisioning in Cloud Computing environment was proposed and the features of this proposal are as follows.

There is some troublesome for users to get user authentication in the existing Cloud Computing environment because they have to go through the user authentication process to use the service every single time by using an ID and authentication method that the service provider provided. So, user authentication platform using Provisioning solves the existing inconvenience and helps use Cloud Computing services easily. 
The proposed platform architecture analyzes user information and authenticates a user through user profile. Also, it has user information stored through user monitoring and there is an advantage that the user authentication process required by the service provider can be omitted when using other Cloud Computing services.

As further study, there should be a study on protection of user information, which is profile and log data in the platform proposed by this paper.

Acknowledgments. This work was supported by the Security Engineering Research Center, granted by the Korea Ministry of Knowledge Economy

\section{References}

1. Lee, T.: Features of Cloud Computing and Offered Service State by Individual Vender. In: Broadcasting and Communication Policy, vol. 22, pp. 18-22 (2000)

2. http://www.gartner.com/technology/symposium/2009/sym19/about.jsp

3. Lee, J., Choi, D.: New trend of IT led by Cloud Computing. In: LG Economic Research Institute, (2010)

4. Lee, H., Chung, M.: Context-Aware Security for Cloud Computing Environment. In: IEEK, vol. 47, pp. 561-568 (2010)

5. Kim, J., Kim, H.: Cloud Computing Industry Trend and Introduction Effect. In: IT Insight, National IT Industry promotion Agency (2010)

6. http://en.wikipedia.org/wiki/Cloud_computing\#History

7. Dikaiakos, M.D., et al.: Cloud Computing Distributed Internet Computing for IT and Scientific Research. In: IEEEInternet Computing, pp. 10-13, September/October (2009)

8. Harauz, J., et al.: Data Security in the World of Cloud Computing. In: IEEE Security \& Privacy, pp. 61-64 (2009)

9. Lee, J.: Cloud Compting, Changes IT Industry Paradigm. In: LG Business Insight, pp. 40-46 (2009)

10. Kim, H., Park, C.: Cloud Computing and Personal Authentication Service. In: KIISC, vol. 20, pp. 11-19 (2010)

11. Armbust, M., et al.: Above the Clouds: A Berkeley View of Cloud Computing. In: Technical Report. http://www.eeec.berkeley.edu/Pubs/TechRpts/2009/EEEC-2009-28.html (2009)

12. Un, S., et al.: Cloud Computing Security Technology. In: ETRI, vol. 24, no. 4, pp. 79-88 (2009) 